\title{
Ovarian Sex Cord-Stromal Tumors in Postmenopausal Women and Total Laparoscopical Management
}

\author{
Andrea Tinelli ${ }^{1}$, Marcello Pellegrino ${ }^{2}$, Vincenzo Emanuele Chiuri ${ }^{3}$, Antonio Malvasi ${ }^{4}$ \\ ${ }^{1}$ Department of Gynecology and Obstetric, Vito Fazzi Hospital, Lecce, Italy; ${ }^{2}$ Department of Pathology, Vito Fazzi Hospital, Lecce, \\ Italy; ${ }^{3}$ Department of Oncology, Vito Fazzi Hospital, Lecce, Italy; ${ }^{4}$ Department of Gynecology and Obstetric, Santa Maria Hospital, \\ Bari, Italy. \\ Email: andreatinelli@gmail.com
}

Received October $26^{\text {th }}, 2009$; revised December $19^{\text {th }}, 2009$; accepted January $28^{\text {th }}, 2010$.

\begin{abstract}
BACKGROUND: Ovarian sex-cord stromal tumors (SCST) take up 5\% of the ovarian neoplasm and may develop into an ovarian mass or a haemoperitoneum. The surgical management of SCST in early-stage adult patients is not well defined. CASE REPORT: A 69 year-old postmenopausal woman was admitted for metrorrhagia, a right ovary mass and increasing pelvic pain. Preoperative clinical and instrumental examination suspected an ovarian tumor, and the laparoscopic right ophorectomy and the frozen section suggested an ovarian SCST. To fast restore and preserve woman integrity, total laparoscopic hysterectomy (TLH) plus left salpingo-ophorectomy (SO) were performed, without complications in the short and long term follow-up. CONCLUSION: In the authors' opinion, the minimally invasive management of SCST by TLH plus bilateral SO followed by a prolonged surveillance and without intensive surgical staging, could be an appropriate clinical and surgical choice in elder patient at early stage, since these tumors are slow at growth, recurring locally and only a long time after initial treatment. We suggest, after a minimally invasive treatment, a possible "wait and see" option, as in our case report.
\end{abstract}

Keywords: Menopause, Laparoscopy, Ovarian Cancer, Sex Cord-Stromal Ovarian Tumors, Granulosa Cell Tumors, Minimally Invasive Treatment.

\section{Introduction}

Ovarian sex-cord stromal tumors represent $5 \%$ of ovarian neoplasm cases, it can occur at any age (mean age early fifties) and generally, they present as an adnexal mass, or a haemoperitoneum, with an estrogenic pattern linked to metrorrhagia, endometrial hyperplasia or adenocarcinoma.

These tumors show a differentiation in sex cordons or in specialized stroma: the latter includes female cellular type (granulosa or theca cell), male cellular type (Sertoli or Leydig cells) and undifferentiated elements.

Cellular variety of sex-cord stromal tumor shows various degrees of differentiation, reproducing a proliferating pattern present in embryogenesis [1].

\section{Pathological Findings of Ovarian Sex-Cord Stromal Tumors (SCST)}

Granulosa cell tumors contain two histotypes: the young type and the adult type; the latter arises frequently after menopause, but it can also occur in puberty, associated with hyperestrogenism in $75 \%$ of total cases, leading to precocious puberty in children and metrorrhagia in adults.

Some of these cases are inactive in hormonal pattern and some are caused by androgen producers [2].

Macroscopically, sex-cord stromal ovarian tumors are capsulated, vary in size and may be solid or partially cystic; the cut surface may be grey-white or yellow, depending on lipid contents; moreover, necrosis and haemorrhage are often present inside the capsule, with cystic compartments filled with fluid or clotted blood.

The microscopic features of sex-cord stromal ovarian tumors may be extremely changeable, even in the same tumor, with a wide variety of patterns and characteristic Call-Exner bodies may be present; they can have in some cases a microfollicular or macrofollicular growth, and in other cases trabecular or insular.

In microscopic diagnosis, cellular elements are often in the shape of coffee beans and, at an immunohysto- 
chemical level sex-cord stromal ovarian tumors express: estradiol or vimentin, inhibin, CD 99 and cytocheratin, with a reactivity to $\mathrm{S} 100$ marker in $50 \%$ of cases $[3,4]$.

Currently, the gold standard treatment for SCST is by surgery, even if its surgical management for early-stage adult patients is not well defined.

\section{Short Report of an Early SCST in Post-Menopausal Women Managed by Laparoscopy}

A 69 year-old woman, para 1 (spontaneous delivery) with an uneventful old gynaecological history and menopause at 51 year's age, was admitted to hospital for genital bleeding and increasing pelvic pain. Clinical examination vaginam exam revealed a palpable right adnexal mass. Transvaginal ultrasound examination revealed sonomorphological uterine fibromatosis with increased endometrial thickness, and a right ovary mass of $8 \mathrm{~cm}$ in diameter, on average, with anechogenic and hypovascularized structure, adherent to uterine posterior wall. The ultrasound exam was confirmed by the Computer Tomography of pelvis and abdomen, performed in contrast, which reported no other diseases or anomalies. Biochemical markers for epithelial or germ cell ovarian tumors did not reveal any anomalies and patients wanted a minimally invasive treatment by laparoscopy. Thus, based on the patient's clinical symptoms and the instrumental examinations, diagnostic laparoscopy was performed, following signed detailed informed consent, based on presurgical findings to rule out malignancy. Laparoscopic access was performed using Direct Visual Access method, at the level of umbilicus, with a $10 \mathrm{~mm}$ diameter optical trocar (Endopath Xcel Bladeless, Ethicon Endo-Surgery, Johnson \& Johnson Company, USA) inserted through an intra umbilical vertical incision. Following the application of carbon dioxide pneumoperitoneum, with intra-abdominal pressure maintained at 15 $\mathrm{mmHg}$, the abdominal cavity was inspected through a zero-degree laparoscope (Karl Storz, Tuttlingen, Germany), connected to a video monitor; three supra-pubic ancillary trocars were placed as follows: one $10 \mathrm{~mm}$ of diameter trocar inserted in the midline, $3 \mathrm{~cm}$ under the umbilicus, and one in each iliac fossa $(5 \mathrm{~mm}$ of diameter on the left side and $5 \mathrm{~mm}$ of diameter on the right size), laterally to inferior epigastric vessels. Before both operative procedures, all pelvic structures were inspected and the abdomen was explored through the laparoscope clockwise. The pelvic-abdominal inspection showed a right ovarian cyst, with a regular surface (Figure 1), some filmy adhesions in the pouch of Douglas and between the posterior uterine wall and right ovary and tube.

The uterus had multiple fibroids and the left ovary was normal; following the patient's decision to receive mini-

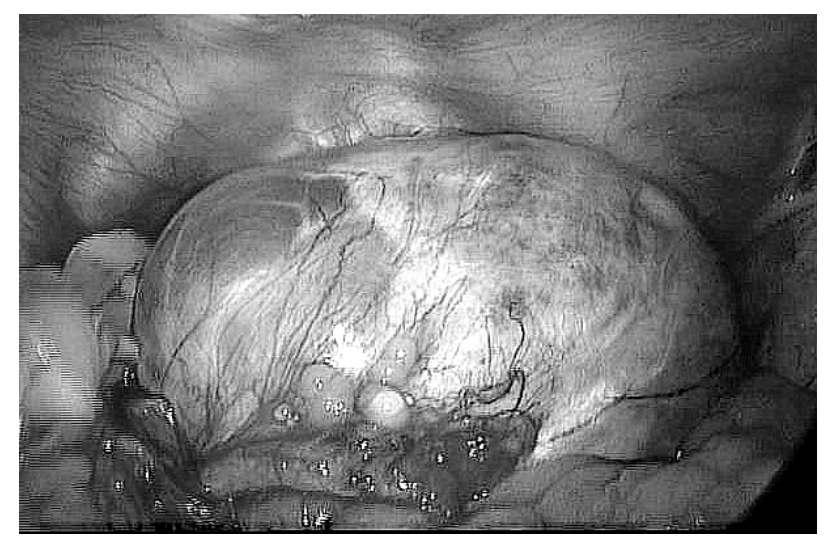

Figture 1. Pelvic-abdominal laparoscopical inspection shows a large right ovarian cyst, with regular white surface, holding Douglas pouch, occluding visualization of the left ovary and uterus

mally invasive treatment (previously signed on informed consent), right ovariectomy was performed by surgeons using a spill-proof endoscopic device (Endobag, Ethicon Endo-Surgery, Johnson \& Johnson Company, USA). In detail, lysis of right ovarian cysts adhesions was performed by aqua dissection with an irrigating probe and, after this, adnexectomy was carried out with a bipolar forceps that coagulated the meso-ovarian vessels, the uterus-ovarian and infundibolopelvic ligaments, while the ovary was sustained by two Manhes forceps. The coagulated ligaments were cut with a monopolar endoscissor and lastly, the tubal wound was irrigated and the haemostasis was re-obtained with fine bipolar scissors. The excised cyst, emptied in Endobag through a laparoscopic sucking-up pin, was removed via one of the trocar through a spill-proof endoscopic device, to avoid accidental leakage of the ovarian cyst content into the peritoneal cavity, which can worsen the prognosis if the cyst turns out to be malignant or can lead to peritonitis if the cyst is a dermoid or mucinous cyst. Following laparoscopy, Dilatation \& Courretage was performed, due to her history of bleeding. The surgical procedure lasted 20 minutes and intra-laparoscopic blood loss was less than $80 \mathrm{cc}$.

The frozen section histology suggested an ovarian SCST, possibly a granulosa cell tumor, while macroscopic examinations showed a smooth right ovarian cortical surface. Following pathologists' advice, in agreement with the patient's preliminary decision and based on her advanced postmenopausal status and slow tumor growth, the surgeons performed a total laparoscopic hysterectomy (TLH) with left salpingo-ophorectomy (SO), which avoided radical treatment by surgical staging, lymphadenectomy, appendectomy and omentectomy.

Preliminarily, the authors inserted in uterus a transvaginal uterine manipulator (Endopath, Ethicon Endosurgery, Inc., Cincinnati, USA) to quickly mobilize the 
uterus during the procedure and activated PK System LYONS Dissecting Forceps and PK System LP Scissors (Gyrus Medical, Inc. Maple Grove, MN, USA). The left broad ligament anterior leaf was opened while the nurse pushed the uterus into retro-version; the left broad ligament posterior leaf was opened to allow the utero-ovarian ligament and tubes to be defined. This step was performed with PK System LYONS dissecting coagulating forceps and PK System LP monopolar scissors, followed by dissection of the same broad ligament. The uterus was pushed by the nurse towards the opposite side, to the right, using uterine manipulator traction; the left uterine vessels were isolated, the left utero-vesical pouch peritoneum was opened by dissecting and grasping coagulating forceps and the bladder was delicately pushed downwards. The surgeons completed the uterine devascularization procedure, after dissecting the left broad ligament: they closed the uterine pedicle with a 0 Vicryl, using a knot pusher after inserting a needle round the uterine vessels. The left uterine vessels were ligated in two places and incised with scissors between the knots, with final haemostasis of the uterine vessels through bipolar forceps, after checking first the position of the ureters. The same actions were performed on the right side of uterus, where the ophorectomy had been previously done. Intra-fascial hysterectomy was continued using the same dissecting coagulating forceps and monopolar scissors, coagulating the cervicovaginal vessels and opening the vagina; anterior and posterior vaginal walls were incised with monopolar scissors and haemostasis was completed through coagulating forceps. After uterine extraction, surgeons performed laparoscopic suturing of the vaginal cuff. The hysterectomy took 65 minutes and the intralaparoscopic blood loss was $200 \mathrm{cc}$. The final examination highlighted: a granulosa cell tumor of right ovary (Figure 2); the uterus showed fibroids and a cystic glandular hyperplasia of endometrium, the left ovary was atrophic.

The FIGO Staging of SCST was IA. The histochemical analysis showed positive reaction to estrogen receptors, with a proliferating index (KI-67-clone MIB1) of $8 \%$ in neoplastic cells. Postoperative recovery was normal, the patient was regularly discharged 3 days after laparoscopy, with no additional therapy at remission and has been followed up for the past three years with instrumental and biochemical monitoring.

\section{Discussion}

In this patient, the surgeons performed a minimally invasive treatment of SCST, avoiding radical treatment by surgical staging with pelvic and para-aortic lymphadenectomy, appendectomy and omentectomy, for the following reasons:

The woman had agreed on minimally invasive treatment, since presurgical assessment did not show ad-

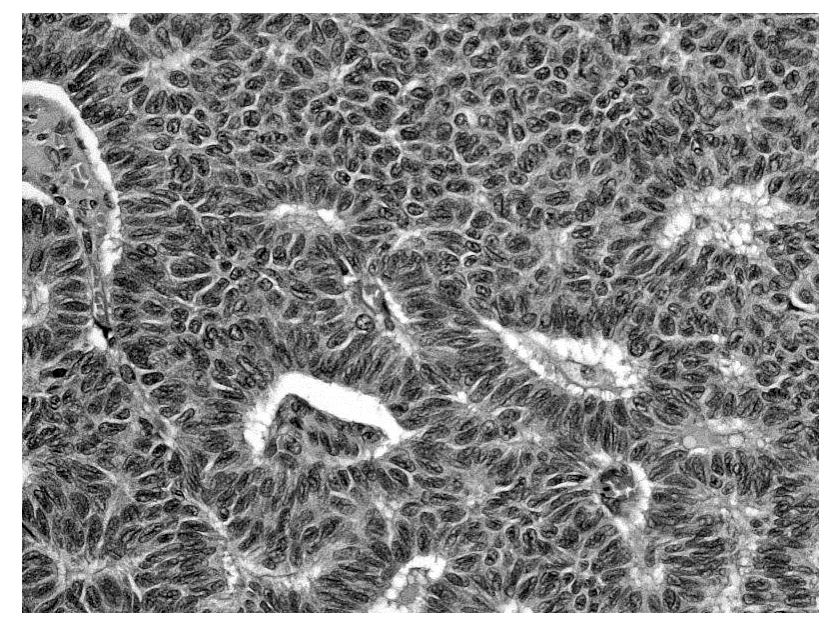

Figture 2. Histology shows adult-type sex cord-stromal tumors of right ovary; the inset shows tumor cells with microfollicular and coffee bean features $(H \& E, \times 20$, original magnification)

vanced malignancy suspect of ovarian cancer; after the frozen section results, surgeons decided the total laparoscopical non-radical unstaged approach either for the patient's advanced postmenopausal age, or for the biology of granulosa ovarian mass-a tumor at slow growing.

The morbidity and morbility of a radical laparoscopic approach, with pelvic and para-aortic lymphadenectomy, appendectomy and omentectomy, collide with the patient wish and with the stage and the biology of the tumor, since more than $95 \%$ of SCST are in first stage, with a mitotic index less than 3 mitosis for $10 \mathrm{HPF}$ in $75 \%$ of cases [5].

A recent study by Brown et al. affirm that lymph node metastasis in ovarian SCST are rare [6].

The stage of disease is the most important prognostic factor associated with the risk of relapse: the 5-year survival rate of stage I ovarian SCST is $86-96 \%$ and all other stages are between $26-46 \%$ [7].

There have been only a few case reports on distant metastases from these tumors $[8,9]$.

Nevertheless, adult types have a better prognosis than young types, and trabecular and follicular histological pattern has a better prognosis than sarcomatoid pattern. Generally, bilateral involvement of the ovaries in SCST is observed in only $2 \%$ of cases, the opposite ovary can be preserved in younger women, while, if the uterus needs to be preserved, endometrium biopsy should be performed because the synchronous occurrence of endometrial adenocarcinoma is associated with estrogen secretion [10].

The differential histological diagnosis of ovarian SCST is with: undifferentiated carcinoma, small cell carcinoma, endometrial stroma sarcoma, cellular fibroid, endometrioid carcinoma with a sex cord-like growing pattern, gonadoblastoma, melanoma or malignant mam- 
malian lobular tumor metastasis [2,3].

The SCST prognosis depends on clinical stage, dimension, cellular atipia presence and tumor rupture [11].

As for recurrence, ovarian SCST can recur long after initial treatment and locally, with an average interval of 5-10 years, with rare metastasis at distance; the longest reported interval is 37 years, therefore lifelong follow-up care is needed [12].

Factors reported to be associated with outcome include: presentation stage, age older than 40 , tumor size, tumor rupture, histologic pattern, high mitotic count, and nuclear atypicality.

The SCST recurrences are peritoneal and retro-peritoneal masses, histologically well expressed [13].

Patients with recurrent disease or residual disease after surgery should be treated with a combination of bleomycin, etoposide, and cisplatin (BEP), while no evidence shows that treatment with progesterone is beneficial and the role of the radiation therapy, especially for palliation in recurrent disease in the pelvis, has yet to be proven $[10,12]$.

A recent article examined the clinical efficacy of bevacizumab with or without concurrent chemotherapy and evaluated the angiogenic characteristics of these tumors; authors concluded that anti-VEGF therapy is highly effective in patients with granulosa cell tumors [13].

For the prolonged surveillance, the tumor marker follow-up of ovarian SCST is through inhibin, a glycoprotein produced by granulosa cells, which may be increased in postmenopausal women with mucinous carcinomas [14].

Concludingly, this short report differs from traditional treatments since the authors propose, for the first time, a minimally invasive treatment in a SCST, for the above reasons.

Primarily, the presurgical assessment did not show advanced malignancy suspect of ovarian cancer and the frozen section results confirmed it. Then, the biology of granulosa ovarian mass, a tumor at slow growing, confirms that lymph node metastasis of SCSTs are rare, so as the early stage of this tumor is the most important prognostic factor associated with the relapse risk, with a survival rate of $86-96 \%$ in stage I(5-year). Nevertheless, adult types have a better prognosis than young types. Finally, the mentioned patient was an elder postmenopausal women of 69 years, so the morbidity and morbility of a more radical laparoscopic approach collided with the patient wish and with the stage and the biology of the tumor.

Given that the literature is lack of laparoscopical treatments of SCST in adult age patients, based on our report, a prospective multicentre trial could be proposed using non-radical laparoscopy in postmenopausal patients with ovarian SCST.

\section{Conclusions}

Generally, SCST has been treated radically in young women [15]; since there are no clear conclusions regarding the role of postoperative chemotherapy or radiotherapy in stage I patients and in those with complete resected tumor [16], TLH plus BSO not followed by lymphadenectomy, has also proven, in our report, to be a safe and effective alternative in postmenopausal patients, with fast recovery and minimal morbidity and morbility. In view of all these findings and due to the technical advances in endoscopic surgery, laparoscopic non-radical treatment of SCST could be considered a safer treatment in selected and consenting adult age patients, which agrees with a minimally invasive surgery and with a successive prolonged surveillance, also defined as "wait and see" approach, since this tumor grows slowly.

\section{REFERENCES}

[1] D. Hasiakos, K. Papakonstantinou, E. Karvouni, and S. Fotiou, "Recurrence of granulosa cell tumor 25 years after initial diagnosis," Report of a case and review of the literature, European Journal Gynaecolology Oncology, Vol. 29, No. 1, pp. 86-88, 2008.

[2] Rosai and Ackerman's surgical pathology, 9th edition, Mosby Ed., Edinburgh, pp. 1691-1694, 2004.

[3] P. B. Clement and R. H. Young, "Atlas of Gynecologic Surgical Pathology, 2nd edition," Saunders Elsevier Ed., New York, USA, pp. 386-391, 2008.

[4] S. Leibl, K. Bodo, M. Gogg-Kammerer, and M Ovarian, "granulosa cell tumors frequently express EGFR (Her-1), Her-3, and Her-4: An immunohistochemical study," Gynecol Oncol, Vol. 101, No. 1, pp. 18-23, 2006.

[5] Y. K. Lee, N. H. Park, J. W. Kim, Y. S. Song, S. B. Kang, and H. P. Lee, "Characteristic of recurrence in adult-type granulosa cell tumor," International Journal of Ginecological Cancer, Vol. 18, No. 4, pp. 642-647, 2008.

[6] J. Brown, A. K. Sood, M. T. Deavers, L. Milojevic, and D. M. Gershenson, "Patterns of metastasis in sex cordstromal tumors of the ovary: Can routine staging lymphadenectomy be omitted?" Gynecologic Oncology, Vol. 113, No. 1, pp. 86-90, 2009.

[7] D. M. Gershenson, "Management of early ovarian cancer: Germ cell and sex cord-stromal tumors," Gynecologic Oncology, Vol. 55, (3 Pt 2), pp. S62-72, 1994.

[8] O. Ismi, Y. Vayisoglu, T. Karabacak, and M. Unal, "Supraclavicular metastases from a sex cord stromal tumor of the ovary," Tumori, Vol. 95, No. 2, pp. 254-257, 2009.

[9] A. Chauhan, P. Dahiya, H. Singh, M. Gill, and S. Verma, "Isolated breast metastasis from granulosa cell tumor of the ovary," Arch Gynecol Obstet, Vol. 280, No. 6, pp. 997-999, December 2009.

[10] N. Colombo, G. Parma, V. Zanagnolo, and A. Insinga, "Management of ovarian stromal cell tumors," Journal of 
Clinical Oncology, Vol. 25, No. 20, pp. 2944-2951, 2007.

[11] A. Auranen, J. Sundström, J. Ijäs, and S. Grènman, "Prognostic factors of ovarian granulosa cell tumor: A study of 35 patients and review of the literature," International Journal of Gynecological Cancer, Vol. 17, No. 5, pp. 1011-1018, 2007.

[12] S. E. Rha, S. N. Oh, S. E. Jung, Y. J. Lee, A. W. Lee, Byun, and J. Y. Recurrent, "Ovarian granulosa cell tumors: Clinical and imaging features," Abdom Imaging, Vol. 33, No. 1, pp. 119-125, 2008.

[13] X. Tao, A. K. Sood, M. T. Deavers, K. M. Schmeler, A. M. Nick, R. L. Coleman, L. Milojevic, D. M. Gershenson, and J. Brown, "Anti-angiogenesis therapy with bevacizumab for patients with ovarian granulosa cell tumors," Gynecology Oncology, Vol. 114, No. 3, pp. 431-436, September 2009.

[14] M. A. Farinola, A. M. Gown, and K. Judson, "Estrogen re- ceptor alpha in ovarian adult granulosa cell tumors and Sertoli-Leydig cell tumors," International Journal of Gynecological Pathology, Vol. 26, No. 4, pp. 375-382, 2007.

[15] G. Hölscher, C. Anthuber, G. Bastert, A. Burges, D. Mayr, E. Oberlechner, G. Schubert-Fritschle, S. Sinz, H. Sommer, B. Schmalfeldt, and J. Engel, "Malignant Ovarian Tumors' of the Munich Cancer Center. Improve- ment of survival in sex cord stromal tumors-an observational study with more than 25 years follow-up," Acta Obstetricia et Gynecologica Scandinavica, Vol. 88, No. 4, pp. 440-448, 2009.

[16] G. V. Koukourakis, V. E. Kouloulias, M. J. Koukourakis, G. A. Zacharias, C. Papadimitriou, K. Mystakidou, K. Pistevou-Gompaki, J. Kouvaris, and A. Gouliamos, "Granulosa cell tumor of the ovary: Tumor review," Integrative Cancer Therapies, Vol. 7, No. 3, pp. 204-215, 2008. 